\title{
A Novel Biometric System based on Hybrid Fusion Speech, Signature and Tongue
}

\author{
Gaganpreet Kaur \\ Research Scholar \\ Punjab Technical University \\ Kapurthala, Punjab
}

\author{
Dheerendra Singh, PhD \\ Professor \& Head \\ SUSCET Tangori \\ Mohali, Punjab
}

\begin{abstract}
There is urgent need to make behavioural biometric systems more reliable, robust and accurate. This paper presents feature level fusion of offline signature and speech and tongue biometrics. The fusion of speech and signature with tongue made the system more efficient. MFCC is used for feature extraction in speech and in signature DCT is used which is widely used for image and texture feature extraction. While the features of tongue are extracted using SIFT algorithm. Hybrid weighted average using Apirori two item set is applied for the fusion of extracted features of all the modalities. SVC2004 signature database are used for experimental results. Tongue database was collected by capturing pictures using digital camera while for speech CMU_ARCTIC database which is available at Language Technologies Institute at Carnegie Mellon University is used in the work. Features for both noisy and non-noisy samples have been collected separately. Gaussian noise was added to the system to check the performance in noisy environment. The proposed system also works efficiently on filtered noisy modalities with accuracy of $80 \%$. The accuracy of the noiseless system is $88.75 \%$ with $0.06 \%$ of FAR and with $0.05 \%$ of FRR and the noisy system have FAR $0.05 \%$ and FRR $0.15 \%$. The ROC curves are calculated.
\end{abstract}

\section{Keywords}

Biometric, Multimodal Biometric, SIFT, Mel Frequency Cepstral Coefficient, Discrete Cosine Transformation, Apriori Algorithm, Weighted Averaging, Motion Blur filter ,False Acceptance Rate, False Rejection Rate, ROC.

\section{INTRODUCTION}

\subsection{What is biometrics?}

Biometrics is a pattern recognition system which has evolved few years back and gained momentum in coming times. Basically, three main families exist in biometric systems (a) Biological (b) Morphological (c) Behavioural [1].Every single biometric system has its own pros and cons. In order to make the system more reliable and accurate various modalities are combined together which lead to the concept of multimodal biometric systems. The multimodal biometric systems provide better performance in terms of false acceptance and false rejection rate. The accuracy of the system also increases manifold.The storage requirements, processing time and overall computational time of a multimodal biometric system are higher than that for a unimodal biometric system [2]. The key processes involved in multimodal biometric system are image acquisition, feature extraction, fusion and matching. The success of multimodal biometric system lies in an effective fusion scheme. Fusion can be performed at various levels (a) Fusion at the data or feature level (b) Fusion at the match score level (c) Fusion at the decision level [3]

\subsection{Weighted Ordered Averaging Function}

Various operators have been used for fusion of different modalities in Biometrics. The common operators developed in mathematics are the sum and the product [4]. These operators have been used together with some other operators like the maximum, median and minimum and the majority voting operator in data fusion[5].Weighted averaging is methods for fusion in which weights are assigned to the features extracted and are calculated as

$$
\mathrm{Q}=\sum_{i=1}^{n} w(\mathbf{i}) * \mathbf{x}(\mathbf{i})
$$

where $w(i)$ is the weight of operator and $x(i)$ is the feature vector extracted.

\subsection{Association Rule Mining}

Association rule mining techniques are used to recognize relationships among a set of items in database [6]. These relationships are based on co-occurrence of the data items. In this context, the association rule mining technique may generate best feature vectors from various biometric modalities which can lead to fusion.

Let $\mathrm{D}$ be a set of transactions, where each transaction $\mathrm{T}$ is a set of items such that $T$ is subset of $I$, where $I=\left\{i_{1}, i_{2} \ldots i_{m}\right\}$ be a set of literals, called items. For a given set of transactions T the problem is to find association rules that have support and confidence greater than the user specified minimum support and minimum confidence [6]. So, support and confidence are measures of the rule. A high value of support indicates that the rule is frequent in the problem. A high value of confidence shows that the rule is true to justify a decision based on it [7].

Support for an association rule is defined as the fraction of transactions in D that contain $\mathrm{X} \cup \mathrm{Y}$. Support (Sup) is calculated by the following formula:

Sup $(X Y)=($ Number of times $X$ and $Y$ appear together $) / D$

where XUY is the number of transactions containing both in $\mathrm{X}$ and $\mathrm{Y}$ and $\mathrm{D}$ is the total number of transactions in the database.

Confidence for an association rule is defined as the fraction of the number of transactions in $\mathrm{N}$ that contain $\mathrm{X}$ also contains Y. Confidence is calculated by the formula:

Confidence $(X Y)=$ Support $(X Y) / S u p p o r t(X) \ldots(2)$

The simplicity of the rule can be defined by the total number of attributes on the antecedent part of the rule i.e. on the left hand side of the rule and tries to calculate the understandability of the rule. Simplicity (Simp) can be calculated by the formula: 
The Complexity of the rule is to make the complex rule simpler if on the consequent part the number of attributes is more. The complexity (Comp) of the rule can be calculated by the formula:

Comp $=1 /(($ Math.log $(1+\mathrm{Z}))+($ Math.log $(1+\mathrm{Y})) /$ (Math.log $(1+$ XUY $)))$

\subsection{Apriori Algorithm}

For finding frequent item sets following steps are followed:

Step 1: Scan all transactions and find all frequent items that have support above s\%. Let these frequent items be P.

Step 2: Build potential sets of $\mathrm{k}$ items from $\mathrm{Pk}-1$ by using pairs of item sets in Pk-1 such that each pair has the first k-2 items in common. Now the k-2 common items and the one remaining item from each of the two item sets are combined to form a k-item set. The set of such potentially frequent $\mathrm{k}$ item sets is the candidate set $\mathrm{CK}$. (For $\mathrm{k}=2$, we build the potential frequent pairs by using the frequent item set L1 appears with every other item in L1. The set so generated is the candidate set $\mathrm{C} 2$.

Step 3: Scan all transactions and find all k-item sets in CK that are frequent. The frequent set so obtained is L2.

The first pass of the Apriori algorithm simply counts item occurrences to determine the large 1-item sets.

A subsequent pass, say pass k, consists of two phases. First, the large item sets $\mathrm{Pk}-1$ found in the $(\mathrm{k}-1)$ th pass are used to generate the candidate item sets $\mathrm{Ck}$, using the apriori- gen function. Next, the database is scanned and the support of candidates in $\mathrm{Ck}$ is counted. For fast counting, there is a need to efficiently determine the candidates in $\mathrm{Ck}$ that are contained in a given transaction $t[8,9]$.

\section{RELATED RESEARCH ON MULTIMODAL BIOMETRIC SYSTEM}

This part of paper focuses more on the state of art on multimodal biometric systems involving various modalities for the fusion.

Biometrics systems, components, requirements and performance of Biometric systems are well explained by Ross, Jain and Delac [10] [12] [11]. They have explained commonly used Biometrics, their limitations, and advantages and compared different Biometrics. They have also explained different types of Biometric systems. Different classifier schemes for fusion are explained by Ben-Yocoub [13] and Prabhakar [14] proposed classifier scheme for classifier combination at decision level. Zanuy [15] explained limitations of unimodal systems and how to overcome those limitations and explained different levels of fusion. Liu [16], Naaz [17] and Diwakar [18] have worked on a unique organ tongue for Biometrics and work in itself is unique. They have explained how to collect samples for tongue and how features can be extracted. Karthik [19] have used face, signature and speech as Biometrics and proposed a multimodal Biometric system using PCA, LDA, DCT, MFCC to extract various features of these modalities. Jain [20] proposed a latent to full palm print matching system. Conti [21] proposed an innovative multimodal Biometric system based on iris and fingerprint traits. Jain [22] proposed a system for matching latent fingerprints found at crime scenes to rolled fingerprints enrolled in law enforcement databases. In addition to minutiae, they also used extended features including singularity, ridge quality map, ridge flow map, ridge wavelength map, and skeleton. Lahane [23] proposed a system using iris and fingerprint as trait in which each trait processes its information independently. A template level fusion algorithm results in a homogeneous Biometric vector by integrating iris and fingerprint data. Kaur [24] [25] [26] [30] [31] have explained different levels of fusion in multimodal Biometric systems in detail. They proposed a new method to fuse offline signatures and speech modalities as these modalities are widely accepted and natural to produce.

\section{TONGUE BIOMETRICS MODEL 3.1 Tongue Biometrics}

Dr. David Zhang of Biometrics Research Centre of Polytechnic University Hong Kong has proved the feasibility of the tongue to be used as Biometrics [27].The human tongue is a unique organ which is difficult to forge and is well protected in the mouth. The tongue presents geometric shape information and physiological texture information which are very useful in identity verification process. Thus, tongue biometric is an extremely reliable means for personal identification. [18].In this work texture feature of the tongue are extracted using Scale Invariant Feature Transform (SIFT) algorithm. Figure 1(a) and (b) represents different shapes of tongue along with textures of tongue.
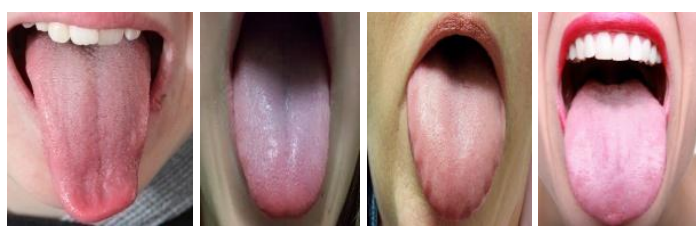

Fig 1: (a) Different shapes of tongue.

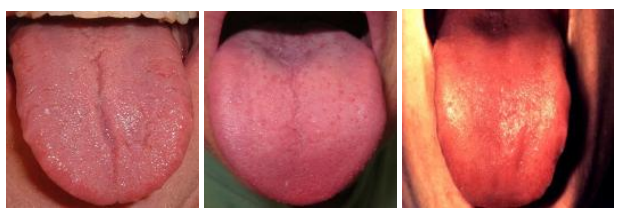

Fig1: (b) Different textures of tongue.

\subsection{Database for Tongue Biometrics}

A digital camera is used for acquiring tongue images. The height and distance of digital camera is always taken into consideration to ensure good clarity and accuracy of tongue images being taken. The images of tongue should be taken in an enclose area to ensure lighting of tongue image in control. In this tongue model 50 images of tongue of different people have been collected and stored in database. Table 3. shows the database images used in the implementation for tongue biometrics.

\subsection{SIFT Algorithm}

Scale invariant feature transform algorithm is a feature vector generation algorithm. SIFT algorithm extracts features of tongue by applying four stage filtering approach which involves:

a) Scale-space extrema detection: Difference of Gaussians is applied for locating scale-space extrema. It searches for all the image locations.

b) Keypoint localization: this stage removes those key points which have low contrast or are poorly localised on an edge. This is achieved by calculating the 
Laplacian value for each keypoint found in a). If the function value is below a threshold value then this point is excluded. This removes extrema with low contrast.

c) Orientation assignment: Orientations are assigned to the keypoints based on local image properties [18].

d) Keypoint Descriptor: The local gradient data is used to measure keypoint descriptors. Then these are transformed and a set of histograms are created over a window centred over keypoint.

\subsection{Tongue Texture Features Extraction}

Tongue texture gives us valuable information about the spatial arrangement of color or intensities in an image or selected region of an image. Features are extracted using SIFT (Scale Invariant Feature Transformation) Normalization is performed using min-max normalization which is also shown in the same figure. Gaussian noise is added to the images. The statistics used to describe the processed tongue images are expressed as follows:

a) Extrema: It specifies the extrema points in the region.

b) Filled area: It specifies the number of on pixels in FilledImage.

c) Solidity: It is computed as Area/ConvexArea

d) Convex area: It specifies the number of pixels in 'ConvexImage'

e) Eccentricity: The eccentricity is the ratio of the distance between the foci of the ellipse and its major axis length. The value is between 0 and 1 .

f) Extent: It specifies the ratio of pixels in the region to pixels in the total bounding box.

The above explained textural features of tongue are computed in Matlab using command: regionprops: which measure the properties of image regions. The command used is $\mathrm{S}=$ regionprops(L, properties) which measures a set of properties for each labeled region in the label matrix $\mathrm{L}$.

\subsection{Implementation Steps of Tongue}

\section{Biometrics}

The following steps were used to implement the Tongue based Biometric Model on Matlab:

Step 1: Collect tongue image sample and convert into gray scale image.
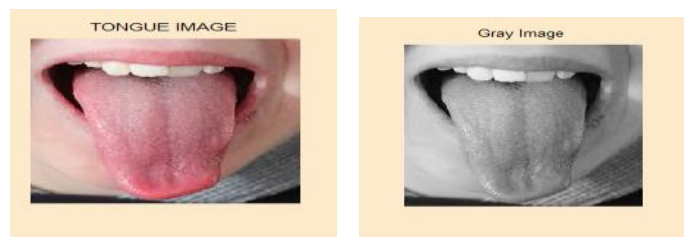

(a)Tongue Image Sample (b) Gray Scale Image

Step 2: Create histogram of processed image.

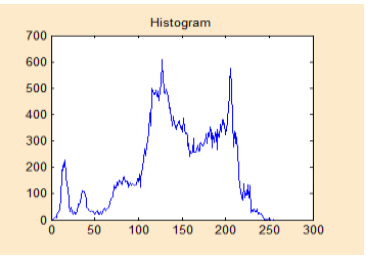

Step 3: Add Gaussian noise to the uploaded image

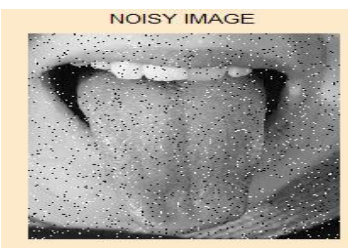

Step 4: Apply motion blur filtering to the noisy image



Step 5: Normalized image of base image

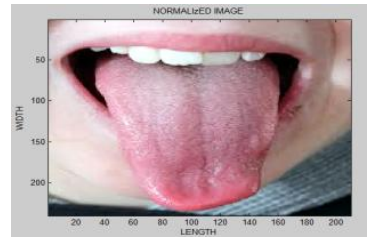

Step 6: Normalized image of noisy image

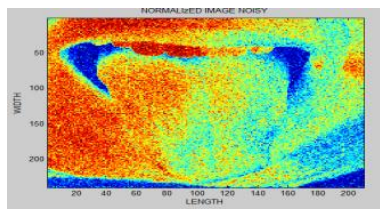

Step 7: Apply SIFT algorithm to find features in the image.


(a) Base image with key points mapped (b) Noisy image with key points mapped

Step 8: Edge image is created 

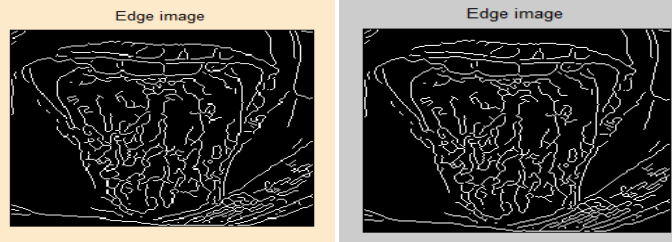

(a) Edge image for non noisy sample (b) Edge image for noisy sample

Step 9: SIFT Image
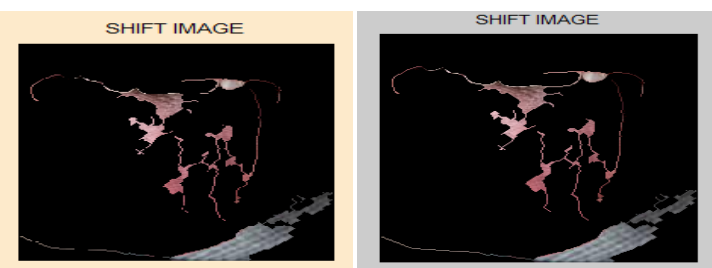

(a) SIFT image for non noisy sample (b) SIFT image for noisy sample

\section{SPEECH BIOMETRICS MODEL}

\subsection{Speech Biometrics}

Speaker recognition is termed as process of recognizing different speakers using their speech [19]. It can be either identification or verification system depending on requirement i.e. to identify the speaker among the group of speakers or verify the identity claim of the speaker. Speech is classified into

a) Text independent

b) Text dependent

Speech Biometrics system is robust and database can be collected with an ease. The feature vectors in speech biometric model are extracted using Mel Frequency Cepstral Coefficients (MFCC).

\subsection{Database for Speech}

The CMU_ARCTIC databases constructed at the Language Technologies Institute at Carnegie Mellon University is used. It is US English single speaker databases which are designed for unit selection speech synthesis research. The databases consist of around 1150 utterances selected. The databases include US English male and female speakers. In this work only male database has been used which contains 1132 utterances spoken by a US English male speaker. Table 3 . shows the database images used in speech biometrics.

\subsection{Mel Frequency Cepstral Coefficient in}

\section{Speech Biometrics}

Mel Frequency Cepstral Coefficient is representation of the spectrum of an audio signal. It takes nonlinear human perception of pitch, which is described by the mel scale. The MFCC algorithm is used for the preprocessing the uploaded speech signal. Gaussian noise has been also added to the speech signal to check the performance of the speech signal. The features have been extracted through a step procedure of MFCC in which features like Hamming distance, Fast Fourier Transformation, MFBP and DCT Quantization has been used.

MFCC is considered as robust, accurate and quite popular method for extracting feature in speech.

\subsection{Speech Features Extraction}

The features used to describe the processed speech samples are expressed as follows:

a) Maximum frequency

b) Minimum frequency

c) Average frequency

d) Rolloff: is defined as the frequency below which $85 \%$ of the magnitude distribution of the spectrum is concentrated.

\subsection{Implementation steps for Speech \\ Biometrics Model}

The following steps were used to implement the Speech based Biometric Model on Matlab:

Step 1: Collect speech signal.

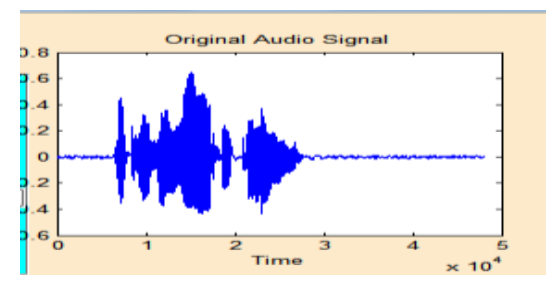

Step 2: Add Gaussian noise to check the performance of the system.

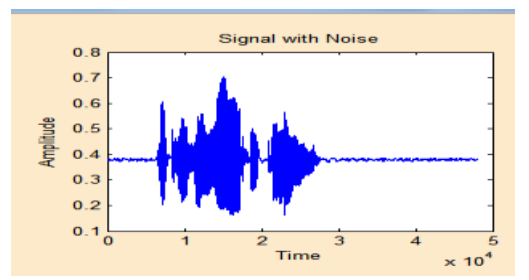

Step 3: Smooth (filtered) signal modified.



Step 4: Hamming window 

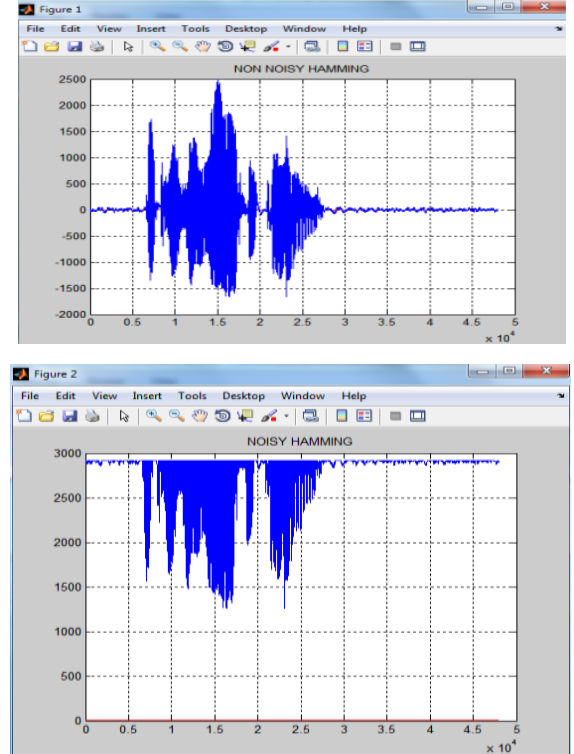

(a) Hamming window for non noisy sample (b) Hamming window for noisy sample

Step: 5 Fast Fourier Transform for noisy and non noisy sample
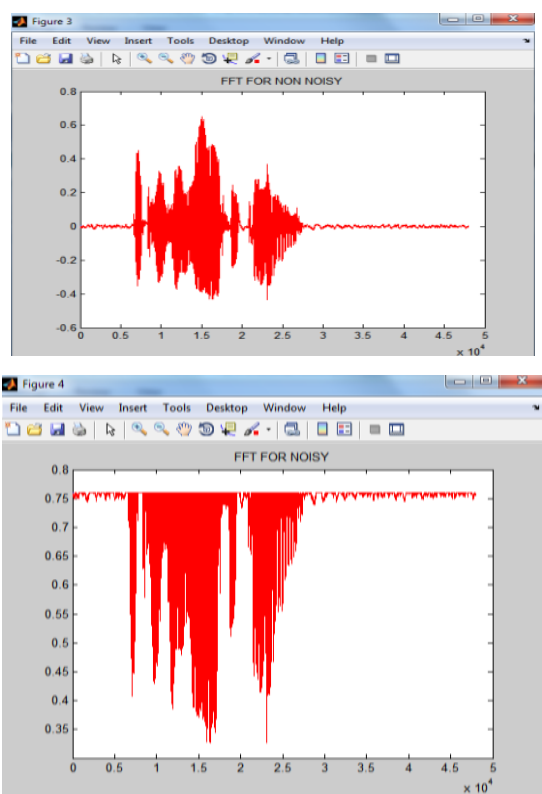

(a) FFT step for non noisy sample (b) FFT step for noisy sample

Step 6: Find MFCC filter-bank for noisy and non noisy sample

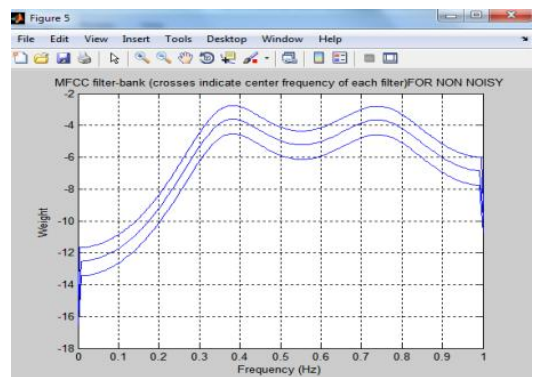

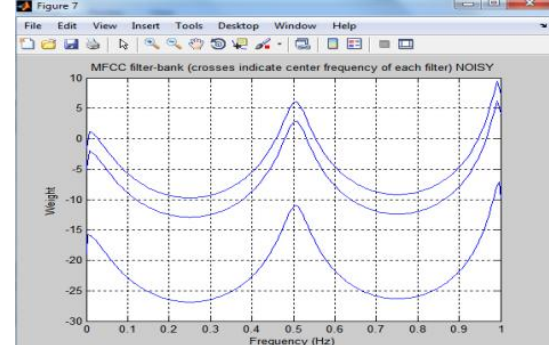

(a) MFCC filter bank for non noisy sample (b) MFCC filter bank for noisy sample

Step 7: Find trajectory graph.


(a) Trajectory for non noisy sample (b) Trajectory for noisy sample

Step 8: Find DCT quantization.


(a) DCT quantization for non noisy sample (b) DCT quantization for noisy sample 


\section{SIGNATURE BIOMETRICS MODEL}

\subsection{Signature Biometrics}

Signature Biometrics is one of the behavioural biometrics which is most legal and common means for identification and verification of user in daily life [29]. How much advancement may come in any field, still for the purpose of authentication in banks, for financial transactions etc. signature system is used. Signature biometrics is split into two main parts:

a) Online/static signature recognition system

b) Offline/dynamic signature recognition system

Signature features are extracted using Discrete Cosine Transform applied on signature database.

\subsection{Database for Signature}

Database used in for the signature biometrics is SVC20. SVC20 is the subset of SVC2004 database. 20 original and 20 forged signatures for 20 signers are there in database. Table 3. shows the database images.

\subsection{Discrete Cosine Transform for Feature Extraction}

Discrete Cosine Transform (DCT) transform is applied to an on-line signature biometrics system to evaluate the features. The discrete cosine transform (DCT) is closely related to the discrete Fourier transform. It is a separable linear transformation. The DCT tends to concentrate information and is useful for image compression applications.

\subsection{Signature Features Extraction}

The extracted features for signature using DCT calculated using regionprops command in Matlab is as follows:

(a)Area: The actual number of pixels in the region.

(b)Extent: It specifies the ratio of pixels in the region to pixels in the total bounding box. It is computed as the Area divided by the area of the bounding box.

(c)Perimeter: the distance around the boundary of the region. regionprops computes the perimeter by calculating the distance between each adjoining pair of pixels around the border of the region.

(d)Centroid: It specifies the center of mass of the region

(e)Total number of black pixels

\subsection{Implementation of Signature Biometrics}

Step 1: Collect signature sample.



Step 2: Convert it into gray scale.

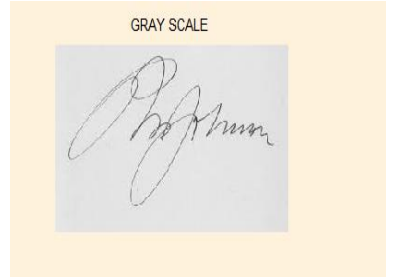

Step 3: Use motion blur filter



Step 4: Noised Signature Sample

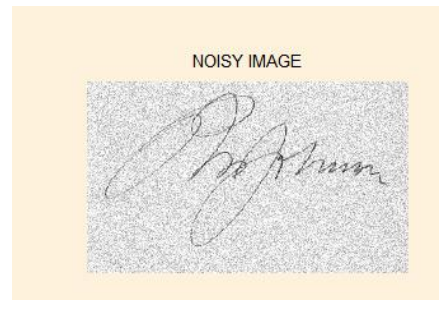

Step 5: Normalized Signature Sample

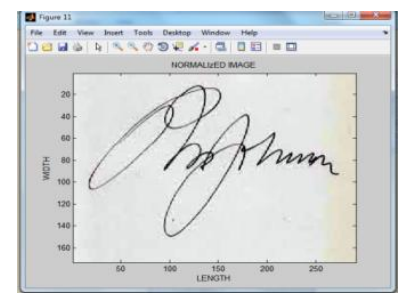

Step 6: Noisy Normalized Signature Sample

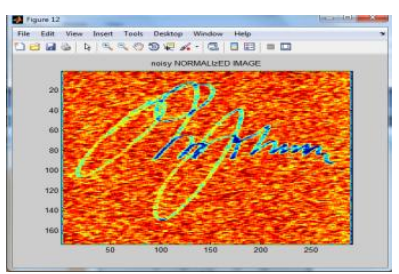

Step 7: Frequency Plot for Signature Sample

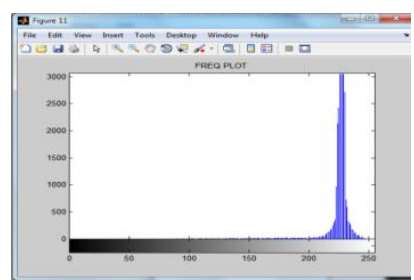


Step 8: DCT applied to Noisy Signature Sample
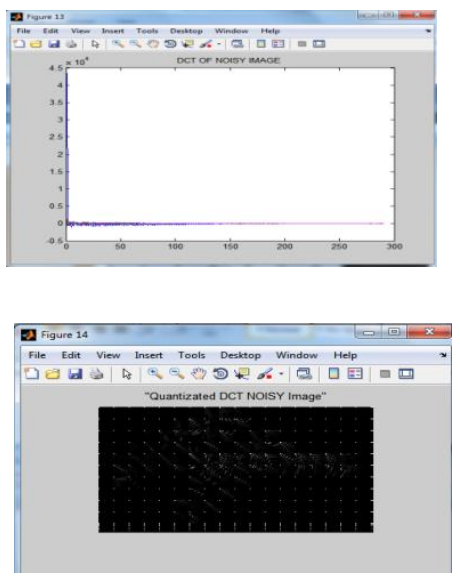

\section{PROPOSED ALGORITHM \& IMPLEMENTATION}

In this paper the fusion is proposed by using new hybrid weighted averaging function with the combination of Support, Confidence, Simplicity and Complexity of apirori algorithm. First the feature vectors for speech, signature and tongue are read. Apriori algorithm is called to calculate two itemsets and to calculate the values of support, confidence, simplicity and complexity. The overall procedure of the proposed algorithm can be described as follows:

Step1: Read the feature vectors for speech $X=\left(x_{1}, X_{2}, \ldots x_{m}\right)$, signature $\mathrm{Y}=\left(\mathrm{y}_{1}, \mathrm{y}_{2}, \ldots \mathrm{y}_{\mathrm{n}}\right.$ and tongue $\mathrm{Z}=\left(\mathrm{z}_{1}, \mathrm{Z}_{2}, \ldots \mathrm{Z}_{\mathrm{p}}\right)$.

Step 2: Initialize $\mathrm{w} 1=3, \mathrm{w} 2=2, \mathrm{w} 3=1, \mathrm{w} 4=1$,

$$
\begin{aligned}
\min \_s u p p & =0, \quad / / \text { Minimum Support } \\
\text { min_confi } & =0.40, \quad / / \text { Minimum Confidence }
\end{aligned}
$$

Step 3: Call apriori algorithm for finding two itemset for feature sets of $\mathrm{X}, \mathrm{Y}, \mathrm{Z}$.

Step 4: Calculate the values of support, confidence, simplicity and complexity by using equation (1), (2), (3) and (4).

Step 5: if $\sup (\mathrm{x})>\min \_$supp

Then store the best feature in database

Step 6: Calculate threshold $($ th $)=w 1+w 2+w 3+w 4$

Step 7: Fusion function using weighted $(\mathrm{F} 1)=$ num $/ \mathrm{den}$

where num $=(\mathrm{w} 1 * \sup +\mathrm{w} 2 * \operatorname{conf}+\mathrm{w} 3 * \operatorname{simp}+\mathrm{w} 4 * \operatorname{comp})$

and den $=($ th $)$

\section{RESULTS}

New hybrid weighted ordered averaging function proposed for the fusion of tongue, speech and signature has been validated by applying the algorithm on vectors generated. The results were calculated on the database in terms of time, false acceptance rate, false rejection rate, accuracy and ROC curves. Figure 2 shows ROC curve generated for the three biometric samples. The performance of the algorithm is analyzed from the time graph (shown in figure 4 and 6) as the time elapsed by non noisy system developed came to be from 20.1 to 23.1 seconds while the fusion time starts from 2.96 to 5 seconds. Similarly, the time taken by noisy samples was from 36.13 to 40.01 seconds and for fusion it took 4.9 to 5 seconds. FAR and FRR of the system and accuracy is shown in Table 1. Table 2 shows time graph calculated for the noisy speech, signature and tongue samples feature extraction and time elapsed for their fusion. Figure 3 and 5, show fusion graph of non noisy and noisy samples. Figure 7 shows the snapshot of features extracted when this work was implemented in Matlab 7.11 (R2010b).

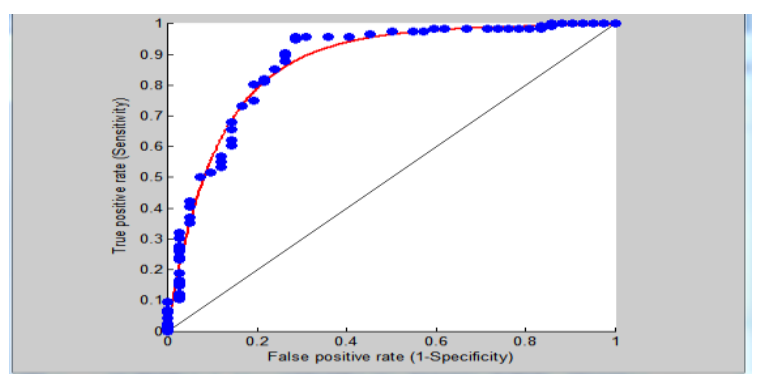

Fig 2: ROC Curve

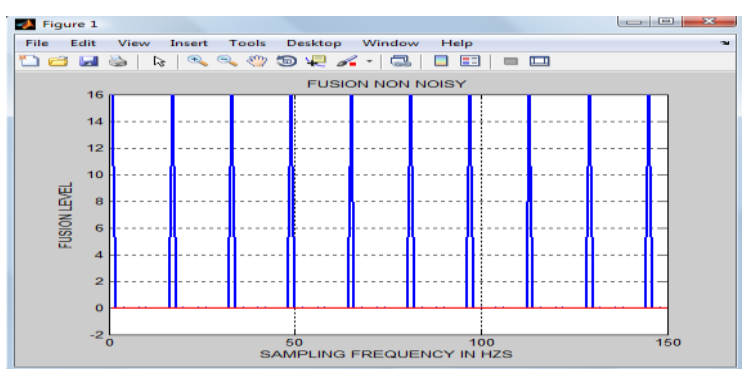

Fig 3: Fusion graph for non noisy samples

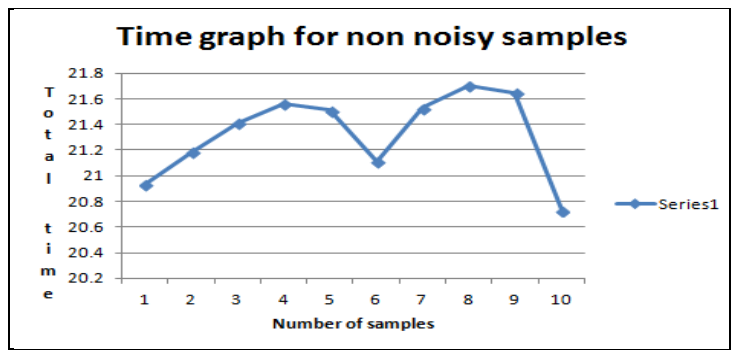

Fig 4: Time Graph for calculated non noisy samples (in seconds)

\section{For non-noisy samples}

False Acceptance Rate $(F A R)=$ Number of falsely accepted samples/Total number of samples

$$
\mathrm{FAR}=5 / 80=0.06 * 100=6.25 \%
$$

False Rejection Rate $($ FRR $)=$ Number of falsely rejected samples/Total number of samples

$$
\mathrm{FRR}=4 / 80=0.05 * 100=5 \%
$$

Accuracy $=(100-($ FAR+FRR $))$

$$
\text { Accuracy }=100-11.25=88.75 \%
$$

\section{For noisy samples}

False Acceptance Rate $(F A R)=$ Number of falsely accepted samples/Total number of samples

$$
\mathrm{FAR}=4 / 80=0.05 * 100 \%=5 \%
$$

False Rejection Rate $($ FRR $)=$ Number of falsely rejected samples/Total number of samples 
$\mathrm{FRR}=12 / 80=0.15 * 100 \%=15 \%$

Accuracy $=(100-($ FAR+FRR $))$

Accuracy $=100-20=80 \%$



Fig 5: Fusion graph for noisy samples

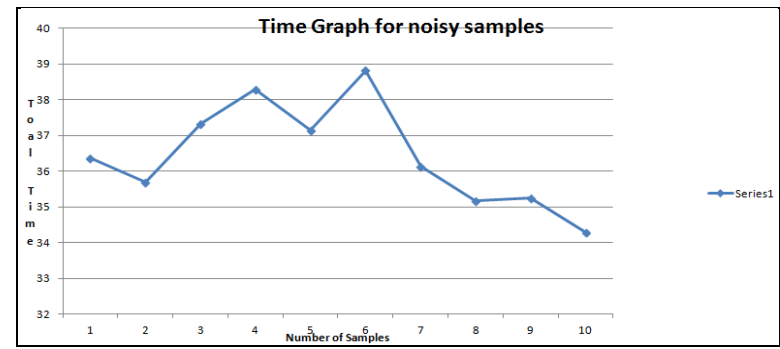

Fig 6: Time Graph for calculated noisy samples (in seconds)

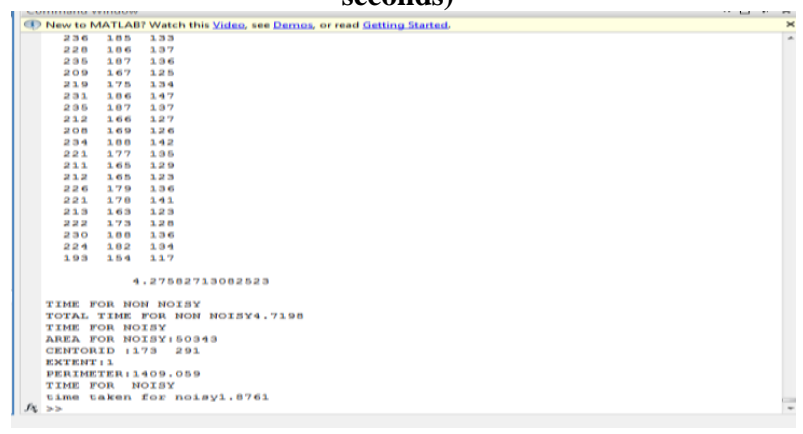

Fig 7: Snapshot showing time taken by noisy and non noisy samples along with values of features extracted in Matlab

Table 1. Accuracy of the work

\begin{tabular}{|l|l|l|l|}
\hline $\begin{array}{l}\text { Multimodal } \\
\text { Biometric System }\end{array}$ & FAR & FRR & Accuracy \\
\hline $\begin{array}{l}\text { For non-noisy } \\
\text { samples }\end{array}$ & 0.06 & 0.05 & $88.75 \%$ \\
\hline For noisy samples & 0.05 & 0.15 & $80 \%$ \\
\hline
\end{tabular}

\section{CONCLUSION AND FUTURE SCOPE}

This work has made use of three biometric speech, signature and tongue modalities to make system more accurate and has also reduced the false acceptance rate and rejection rate by their fusion using modified weighted averaging function. The work has been researched in noisy and non-noisy environment to know the accuracy. ROC curves were calculated. The total time elapsed by the system in each phase was also calculated independently. The system has shown enthusiastic results in both the environments. The new hybrid algorithm worked efficiently for fusion.
The future scope could be further to work on making system more secure and accurate by increasing database.

\section{REFERENCES}

[1] Giot, R., El-Abed, M., Rosenberger, C., "Fast Learning for Multibiometrics Systems using Genetic Algorithms" published in International Conference on High Performance Computing and Simulation (HPCS), Caen, France, $\quad$ pp. $266 \quad-\quad 273, \mathrm{DOI}$ : 10.1109/HPCS.2010.5547127,2010

[2] Md. Maruf Monwar and Marina L. Gavrilova, "Multimodal Biometric System Using Rank-Level Fusion Approach", IEEE Transactions on Systems, Man, and Cybernetics-Part B: Cybernetics, Vol. 39, No. 4, August 2009.

[3] Ross, A., and Jain, A.K., "Multimodal Biometrics: An Overview", published in proc. of 12th European Signal Processing Conference (EUSIPCO), Vienna, Austria, pp. 1221-1224, September 2004. [4] Aureli Soria-Frisch, Alejandro Riera, Stephen Dune, "Fusion Operators for Multi-Modal Biometric Authentication Based On Physiological Signals".

[5] Kittler, J., Hatef, M., Duin, R. P. W., and Matas, J., "On Combining Classifiers," Pattern Analysis and Machine Intelligence, IEEE Transactions on, Vol. 20, No. 3, pp. 226-239, 1998.

[6] Margahny, M. H., and Mitwaly, A. A., "Fast Algorithms for Mining Association Rules," published in conference proceedings AIML 05, Cairo, Egypt, pp. 19-21, December 2005.

[7] Boullé, M., "Khiops: A Statistical Discretization Method of Continuous Attributes", published in Journal of Machine Learning, Vol. No. 55, 2004.

[8] Agrawal, R., Imielinksi, T., and Swami, A., "Mining association rules between sets of items in large databases", in proc. of the ACM SIGMOD Conference on Management of Data, pp. 207-216, 1993.

[9] Gupta, G.K., "Introduction to Data Mining with Case Studies”, Prentice Hall India, 2006.

[10] Ross, A., and Jain, A., "Information Fusion in Biometrics", Pattern Recognition Letters, Vol. 24, No. 13, pp. 2115-2125, 2003.

[11] Delac, K., and Grgic, M., "A Survey of Biometric Recognition Methods", in 46th International Symposium Electronics in Marine, ELMAR-2004, pp. 184-193, 2004.

[12] Jain, A.K., Ross, A., and Prabhakar, S., "An Introduction to Biometric Recognition", IEEE Transactions on Circuits and Systems for Video Technology, Vol. 14, No. 1, pp. 1782-1793, 2004.

[13] Yaoub, S. B., Abdeljaoued, Y., and Mayoraz, E., "Fusion of Face and Speech data for Person Identity Verification", IEEE Transactions on Neural Networks, Vol. 10, No. 5, pp. 1065-1074, 1999.

[14] Prabhakar, S., and Jain, A., "Decision-level in Fingerprint Verification", Pattern Recognition, Vol. 35, No. 4, pp. 861-874, 2001. 
[15] Zanuy, M.F., "Data Fusion in Biometrics", IEEE A \& E Systems Magazine, pp.34-38, 2005.

[16] Liu, Z., Yan, J.Q. , Zhang, D., and Tang, Q.L., "A Tongue-Print Image Database For Recognition", in Proceedings of the Sixth International Conference on Machine Learning and Cybernetics, Hong Kong, pp. 1922,2007 .

[17] Naaz, R., Kundra, S., Garg, P., and Sharma, A., "Tongue Biometric and its Application in Public Use System", IEEE 3rd International Conference on Machine Learning and Computing, Vol. 4, pp. 185-188, 2011.

[18] Diwakar, M., and Maharishi, M., "An Extraction and Recognition of Tongue-Print Images for Biometrics Authentication System", International Journal of Computer Applications, Vol. 61, No. 3, pp. 36-42, 2013.

[19] Karthik, P., Prasad, R.V.S.S., and Prasanna, S.R.M., "Noise Robust Multimodal Biometric Person Authentication System using Face, Speech and Signature Features" , in India Conference, 2008, INDICON 2008, Annual IEEE, pp.23-27, 2008.

[20] Jain, A. K., and Feng, J., "Latent Palmprint Matching", IEEE Transactions on Pattern Analysis and Machine Intelligence, Vol. 31, No. 6, pp. 1032-1047, 2009.

[21] Conti, V., MiliTello, C., Sorbello, F., and Vitabile, S., "A Frequency-based Approach for Features Fusion in Fingerprint and Iris Multimodal Biometric Identification Systems", IEEE Transactions on Systems, Man, and Cybernetics-Part C: Applications and Reviews, Vol. 40, No.4, pp. 384-395, 2010.

[22] Jain, A.K., and Feng, J., "Latent Fingerprint Matching", IEEE Transactions on Pattern Analysis and Machine Intelligence, Vol. 33, No. 1, pp. 88-100, 2011.

[23] Lohane, P. U., and Ganorkar, S. R., "Efficient Iris and Fingerprint Fusion for Person Identification",
International Journal of Computer Applications, Vol. 50, No. 17, 2012.

[24] Kaur, D., Kaur, G., and Singh, D., "Efficient and Robust Multimodal Biometric System for Feature Level Fusion (Speech and Signature)", International Journal Of Computer Applications, Vol. 75, No. 5, pp. 33-38, 2013.

[25] Kaur, D., and Kaur, G., "Level of Fusion in Multimodal Biometrics: a Review", International Journal of Advanced Research in Computer Science and Software Engineering, Vol. 3, Issue 2, pp. 242-246, 2013.

[26] Kaur, D., and Kaur, G., "Feature Level Fusion of Multimodal Biometrics for Personal Authentication", International Journal of Computers \& Technology, Vol. 4, No. 2, 2013.

[27] Mohammad Reza Shahriari,Shirin Manafi,Sepehr Sadeghian, "A Novel Biometric System Based on Tongue Print Images", in International Conference on Information Warfare and Security, pp.22-23,ISBN No. 9781908272782,2012.

[29] S.A. Angadi, Smita Gour, Gayatri Bajantri, "Offline Signature Recognition System using Radon Transform", at IEEE, Fifth International Conference on Signal and Image processing, 2014.

[30]Kaur, G., Singh, D., Kaur, S., "Pollination Based Optimization for Feature Reduction at Feature Level Fusion of Speech \& Signature Biometrics", at IEEE, pp. 1-6, DOI: 10.1109/ICRITO.2014.7014771.

[31] Kaur, G., Kaur, D., and Singh, D., "Enhancement of Security using Feature Level Fusion for Multimodal Biometrics for Speech \& Signature", Published in Elsevier, pp. 25-28, ISBN No. 9789351071624.

[32] Paulino, A.A., Feng, J., and Jain, A. K., "Latent Fingerprint Matching Using Descriptor-Based Hough Transform", IEEE Transactions on Information Forensics and Security, Vol. 8, No. 1, pp. 31-45, 2013.

Table 2. Time Elapsed (seconds) by the noisy samples (in feature extraction, fusion)

\begin{tabular}{|c|c|c|c|c|c|}
\hline $\begin{array}{l}\text { Sample } \\
\text { No. }\end{array}$ & Speech Sample & Tongue Sample & Signature Sample & Fusion & Total Time \\
\hline 1. & 4.67 & 11.66 & 1.82 & 4.34 & 22.49 \\
\hline 2. & 5.11 & 13.53 & 2.18 & 4.28 & 25.1 \\
\hline 3. & 4.33 & 11.45 & 2.29 & 4.12 & 22.19 \\
\hline 4. & 4.32 & 11.08 & 1.83 & 3.96 & 21.19 \\
\hline 5. & 4.31 & 11.52 & 2.6 & 4.01 & 22.44 \\
\hline 6. & 4.37 & 9.82 & 2.7 & 4.04 & 20.93 \\
\hline 7. & 4.35 & 10.61 & 2.42 & 4.03 & 21.41 \\
\hline 8. & 4.46 & 10.64 & 2.29 & 4.17 & 21.56 \\
\hline 9. & 4.7 & 12.02 & 2.1 & 4.16 & 22.98 \\
\hline 10. & 4.36 & 11.3 & 2.63 & 4.31 & 22.6 \\
\hline 11. & 4.51 & 11.36 & 1.98 & 4.48 & 22.33 \\
\hline 12. & 4.62 & 9.92 & 2.73 & 4.24 & 21.51 \\
\hline
\end{tabular}




\begin{tabular}{|c|c|c|c|c|c|}
\hline 13. & 4.57 & 10.84 & 1.96 & 4.3 & 21.67 \\
\hline 14. & 4.56 & 10.16 & 1.7 & 5.11 & 21.53 \\
\hline 15. & 5.7 & 10.88 & 1.75 & 4.12 & 22.45 \\
\hline 16. & 4.31 & 10.93 & 2.2 & 4.26 & 21.7 \\
\hline 17. & 4.52 & 11.28 & 1.72 & 4.13 & 21.65 \\
\hline 18. & 4.47 & 10.14 & 2 & 4.12 & 20.73 \\
\hline 19. & 4.37 & 10.95 & 3.34 & 4.41 & 23.07 \\
\hline 20. & 4.37 & 11.27 & 2.8 & 4.22 & 22.66 \\
\hline 21. & 4.38 & 11.82 & 2.21 & 4.51 & 22.92 \\
\hline 22. & 4.42 & 10.39 & 1.61 & 4.69 & 21.11 \\
\hline 23. & 4.31 & 11.02 & 2.56 & 4.54 & 22.43 \\
\hline 24. & 4.28 & 11.09 & 2.67 & 4.55 & 22.59 \\
\hline
\end{tabular}

Table 3. Test Images

a) Tongue Database

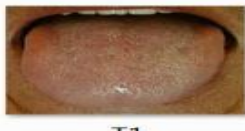

T1

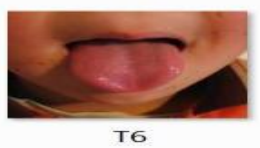

b) Signature Database [SVC 20]

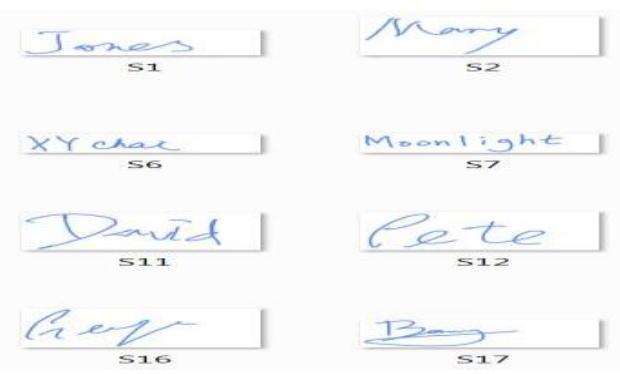

c) Speech Database [CMU_ARCTIC]
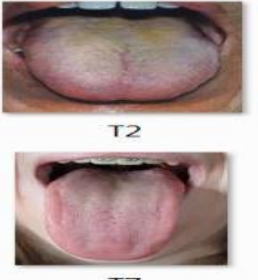

T7

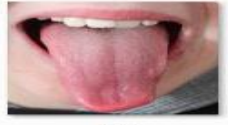

T3

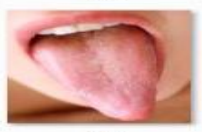

T8


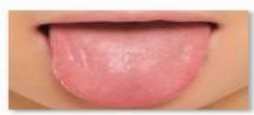

T5

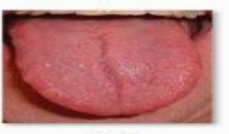

T10

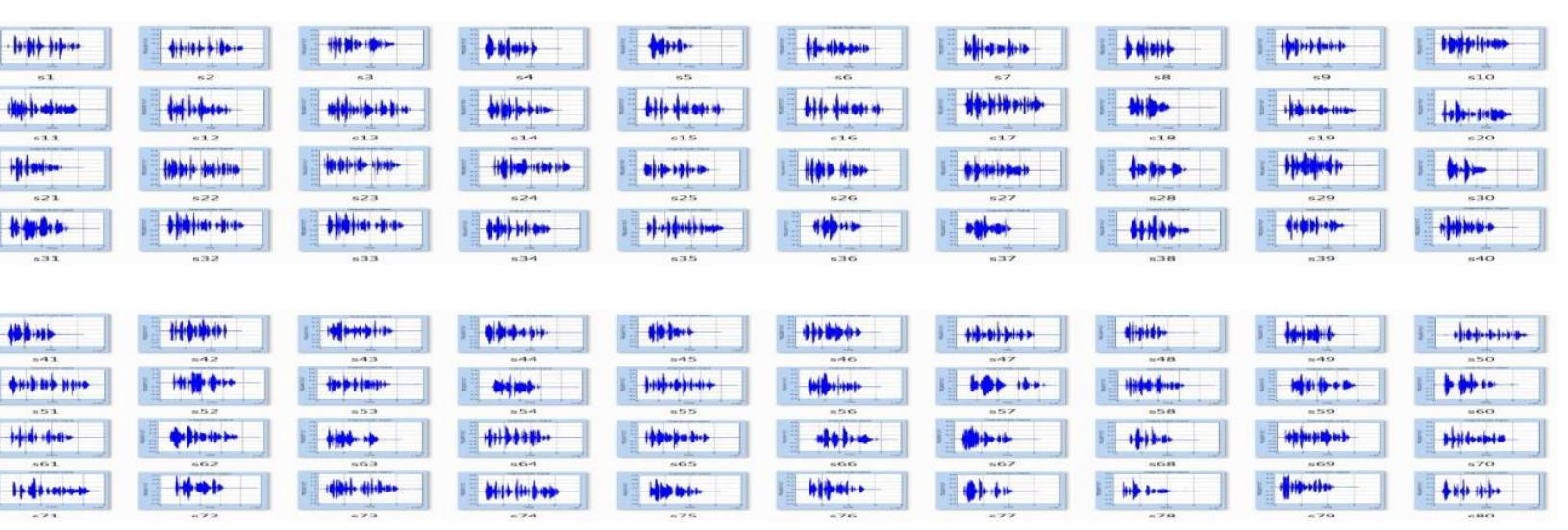

